\title{
Effect of external stress on $\gamma$ nucleation and evolution in TiAl alloys
}

\author{
C. Y. Teng ${ }^{\text {a, b, c }}$, A. Du ${ }^{\text {a }}$, D. S. Xu ${ }^{\text {b, }{ }^{*}}$, Y. Wang ${ }^{c, ~}{ }^{*}$ and R. Yang ${ }^{b}$ \\ ${ }^{a}$ College of Sciences, Northeastern University, 3 Wenhua Road, Shenyang 110004, China \\ ${ }^{\mathrm{b}}$ Institute of Metal Research, Chinese Academy of Sciences, 72 Wenhua Road, Shenyang
} 110016, China

${ }^{\mathrm{c}}$ Department of Materials Science and Engineering, The Ohio State University, 2041 College

Road, Columbus, OH 43210, USA

* Corresponding author. E-mail: dsxu@imr.ac.cn (D. S. Xu); wang.363@osu.edu (Y. Wang)

\begin{abstract}
We have shown in a previous paper that twin boundary area fraction in lamellar $\gamma$-TiAl based alloys depends on collective or correlated nucleation induced by long-range elastic interactions among nucleating precipitates or between pre-existing and nucleating precipitates. In this study we investigate the effects of applied stresses on $\gamma$ nucleation and evolution by elastic interaction energy calculations and phase field simulations. It is found that the elastic interaction energy reaches minimum when two variants are twin related because of their self-accommodation of the coherency strain. When external stresses are present, such autocatalysis during nucleation could be either enhanced or suppressed. Compression perpendicular or tension parallel to the basal plane of the $\alpha$ phase is found to enhance the internal elastic interactions between nucleating precipitates, promote twin variant selection and reduce the overall nucleation rate, while isostatic pressing, compression parallel, tension perpendicular to the basal plane does the opposite. Shear along the interface
\end{abstract}


always reduce the twin boundary area fraction, regardless of the shear direction. These findings could shed light on optimizing processing routes to increase twin boundary area fraction in lamellar microstructures of $\gamma$-TiAl based alloys.

Keywords: A. Intermetallics; B. Phase transformation; D. Microstructure; E. Phase field modeling;

\section{Introduction}

The mechanical properties of a fully lamellar structure depend not only on the grain size $[1,2]$ and texture $[3,4]$, which related strongly to the size and orientation of $\gamma+\alpha_{2}$ lamellae, but also on the fractions of different types of interfaces in the lamellae such as twin, pseudo-twin and ordered domain boundaries between $\gamma$ lamellae and also $\gamma / \alpha_{2}$ interfaces [5], therefore the lamellar microstructure control is a key to obtaining balanced mechanical properties of $\gamma$-TiAl based alloys. The twin boundaries have been found beneficial to the room temperature ductility [6] and high temperature microstructure stability [5] of TiAl alloys. Our previous studies [7] have shown that in the absence of external stresses, the frequency of twin boundary depends on whether the nucleation is independent, collective or correlated as a result of the interplay among several factors including long-range elastic interactions between nucleating precipitates or between pre-existing and nucleating precipitates. However, certain external stress exists in many circumstances during phase transformation. First, for many applications, thermal mechanical processing such as extrusion $[8,9]$ or rolling $[10,11]$ is needed as steps to form the parts and optimize the properties of the alloy. 
Under such condition, the $\alpha$ to $\gamma$ phase transformation may take place under external stresses of various type. Second, in the cases of components made from powder metallurgy (PM) or casting, the hot isostatic pressing (HIPping) is often a necessary step for shape formation and defect elimination, the microstructure may also be altered by adjusting temperature and pressure during or after HIPping [12]. Third, the applied stress could influence the microstructure stability during service such as under high temperature creep [13]. However, it is unclear how external stresses affect the frequency of various types of lamellar boundaries under these conditions.

In the present paper, we study the effect of external stresses on the nucleation and growth of $\gamma$ precipitates and analyze the change of lamellar boundary area fraction as a function of the magnitude and type of applied stresses using the phase field method [14-18]. The nucleation and growth of precipitates under different stress conditions including tension, compression, isostatic pressing and shear are simulated using the phase field method. And then the elastic interaction energies between a pre-existing and nucleating precipitates are calculated by using phase field microelasticity theory. The fractions of various types of interfaces are obtained from the simulation and analyzed with the help of the interaction energy calculation.

2. Orientation relationship and phase field formulation of $\alpha \rightarrow \gamma+\alpha_{2}$ phase transformation in TiAl

For Ti-Al alloys, within the $\mathrm{Al}$ concentration range of $39-46.5$ at. $\%$, the phase transformation path is assumed to be $\alpha \rightarrow \gamma+\alpha_{2}$ [19]. The $\alpha$ phase has HCP structure, 
and will order into an HCP based $\alpha_{2}$ phase at lower temperature, and the $\gamma(\mathrm{TiAl})$ and $\alpha_{2}\left(\mathrm{Ti}_{3} \mathrm{Al}\right)$ phases have $\mathrm{L}_{0}$ and $\mathrm{D}_{19}$ crystal structures, respectively. The two phases follow the Blackburn orientation relationship (OR) [20]: $(0001)_{\alpha_{2}} / /\{111\}_{\gamma},<11 \overline{2}$ $0>_{\alpha_{2}} / /<1 \overline{1} 0>_{\gamma}$. In this study, we define the coordinate system using the $\alpha_{2}$ phase as a reference, i.e., $\mathrm{X}[11 \overline{2} 0], \mathrm{Y}[\overline{1} 100]$ and $\mathrm{Z}$ [0001]. Thus the six $\gamma$ variants can be formed by the transformation shear with respect to the basal plane of the $\alpha_{2}$ phase, e.g., variant one $\left(\mathrm{V}_{1}\right)$ corresponds to a shear along the positive direction of $\mathrm{Y}$ and its twin related variant $\mathrm{V}_{2}$ corresponds to a shear along the negative direction of $\mathrm{Y}$. The twin pair will be described as $\mathrm{V}_{1}-0^{\circ} / \mathrm{V}_{2}-180^{\circ}$ hereafter, where the angles represent the shear direction for $\gamma$ variants forming measured as the angles of rotations around the $\mathrm{Z}$ axis, starting from the $\mathrm{Y}$ axis. Similarly, the other twin pairs are $\mathrm{V}_{3}-120^{\circ} / \mathrm{V}_{4}-300^{\circ}$ and $\mathrm{V}_{5}-240^{\circ} / \mathrm{V}_{6}-60^{\circ}$. Three orientation relationships will be formed between different neighboring $\gamma$ variants from such transformation, and three types of lamellar boundaries are formed accordingly, i.e., twin (T), pseudo-twin (PT) and ordered-domain (OD) boundaries. The interfaces formed from different variants are listed in Table 1. The stacking sequence ABABAB of HCP becomes either ABCABC or ACBACB through shear during phase transformation. Group $\gamma_{\mathrm{I}}$ and $\gamma_{\mathrm{II}}$ represent $\mathrm{ABC}$ and $\mathrm{ACB}$ stacking sequence, respectively. The $\mathrm{V}_{1}, \mathrm{~V}_{3}$ and $\mathrm{V}_{5}$ form group $\gamma_{\mathrm{I}}$, while the other variants belong to group $\gamma_{\mathrm{II}}$. The misorientation angles of the pseudo-twin, ordered-domain and twin related variants are $60^{\circ}, 120^{\circ}$ and $180^{\circ}$, respectively.

In the phase field model of $\alpha \rightarrow \gamma+\alpha_{2}$ transformation in TiAl alloys [7], an arbitrary 
microstructure is characterized by introducing six structural order parameter fields and one concentration field. Within the composition and temperature range of our simulation, the $\alpha$ and $\alpha_{2}$ phases have similar Gibbs free energy, and they also have similar crystal structure and lattice parameter. Then it is not necessary to distinguish them by the order parameters, for simplicity, we use one set of structural order parameters to describe $\alpha / \alpha_{2}$. The order parameters 0 or 1 represent the $\alpha / \alpha_{2}$ or $\gamma$ phase, respectively. The evolution of the microstructure is described by the time-evolution of the structural order parameter fields and concentration field following the time-dependent Ginzburg-Landau equation and the non-linear Cahn-Hilliard diffusion equation, respectively, i.e.,

$$
\begin{gathered}
\frac{\partial \eta_{p}(\mathrm{r}, \tau)}{\partial \tau}=-\left[\frac{\partial f_{a}}{\partial \eta_{p}(\mathrm{r}, \tau)}-\sum_{i, j=1}^{3} \beta_{i j}^{p} \frac{\partial^{2} \eta_{p}(\mathrm{r}, \tau)}{\partial u_{i} \partial u_{j}}+\phi \frac{\delta E_{\mathrm{el}}}{\delta \eta_{p}(\mathrm{r}, \tau)}\right]+\xi_{p}(\mathrm{r}, \tau) \\
\frac{\partial c(\mathrm{r}, \tau)}{\partial \tau}=v \nabla^{2}\left[\frac{\partial f_{a}}{\partial c(\mathrm{r}, \tau)}-\alpha \nabla^{2} c(\mathrm{r}, \tau)\right]+\zeta(\mathrm{r}, \tau)
\end{gathered}
$$

where $\xi_{\mathrm{p}}(\mathbf{r}, \tau)$ and $\zeta(\mathbf{r}, \tau)$ are the random noise terms related to fluctuations in the structural order parameter and concentration, respectively.

$$
f_{a}\left(c, \eta_{1}, \eta_{2}, \cdots, \eta_{6}\right)=\frac{a_{1}}{2}\left(c-c_{1}\right)^{2}+\frac{a_{2}}{2}\left(c_{2}-c\right) \sum_{p=1}^{6} \eta_{p}^{2}-\frac{a_{3}}{4} \sum_{p=1}^{6} \eta_{p}^{4}+\frac{a_{4}}{6}\left(\sum_{p=1}^{6} \eta_{p}^{2}\right)^{3}
$$

is the local chemical free energy [21, 22], $a_{1}$ to $a_{4}$ are constants determined by the difference $\Delta f$ of the free energy from Pandat shown in Fig. 2(b).

$$
\begin{aligned}
& E_{\mathrm{el}}=\frac{V}{2} C_{i j k l} \bar{\varepsilon}_{i j} \bar{\varepsilon}_{k l}-V C_{i j k l} \bar{\varepsilon}_{i j} \sum_{p=1}^{6} \varepsilon_{k l}^{p 0} \overline{\eta_{p}^{2}(\mathrm{r})} \\
& +\frac{V}{2} C_{i j k l} \sum_{p, q=1}^{6} \varepsilon_{i j}^{p 0} \varepsilon_{k l}^{q 0} \overline{\eta_{p}^{2}(\mathrm{r}) \eta_{q}^{2}(\mathrm{r})} \\
& -\frac{1}{2} \sum_{p, q=1}^{6} \oint_{\frac{d^{3} \mathrm{~g}}{(2 \pi)^{3}} B_{p q}(\mathbf{n})\left\{\eta_{p}^{2}(\mathrm{r})\right\}_{g}^{*}\left\{\eta_{q}^{2}(\mathrm{r})\right\}_{g}} \\
& -V \sigma_{i j}^{e x} \bar{\varepsilon}_{i j}
\end{aligned}
$$


is the elastic energy under an applied stress, $\sigma_{i j}^{e x}$, following the microelasticity theory for multi-phase coherent solids originated from Khachaturyan [23-25]. In the above equations $\overline{(\ldots)}$ represents the volume average of $(\ldots), V$ is the total volume of the system, $C_{i j k l}$ is the elastic moduli tensor, $\varepsilon_{i j}$ is the elastic strain tensor, $\varepsilon_{i j}^{p 0}$ is the stress-free transformation strain tensor of variant $p$ of the $\gamma$-phase when the corresponding order parameter assumes its equilibrium value, the same as that in reference [21]. $B_{p q}(\mathbf{n})$ is a two-body elastic interaction potential given by

$$
B_{p q}(\mathbf{n})=n_{i} \sigma_{i j}^{p 0} \Omega_{j k}(\mathbf{n}) \sigma_{k l}^{p 0} n_{l}
$$

where $\mathbf{n}=\mathbf{g} / \mathrm{g}$ is a unit vector in reciprocal space and $n_{i}$ is its $i$ th component, $\sigma_{i j}^{p 0}=$ $C_{i j k l E_{k l}^{p 0}}$ and $\Omega_{i j}(\mathbf{n})$ is a Green function tensor which is inverse to the tensor

$$
\Omega_{i j}^{-1}(\mathbf{n})=C_{i k l j} n_{k} n_{l}
$$

The elastic interaction between pre-existing and nucleating variants can be expressed as $[16-18,23]$ :

$$
\Delta E_{\mathrm{el}}=\sum_{p} \sum_{q} \int \frac{\mathrm{dg}}{(2 \pi)^{3}} B_{p q}(\mathbf{n})\left\{\eta_{p}(\mathrm{r})\right\}_{g}\left\{\Delta \eta_{q}(\mathrm{r})\right\}_{g}^{*}
$$

where $\Delta \eta_{q}(\mathrm{r})$ is the change in the profile of the $q$ th structural order parameter field associated with the nucleation events, and $\left\{\Delta \eta_{q}(\mathrm{r})\right\}_{g}^{*}$ is its Fourier transformation.

The parameters $\phi, \beta_{i j}^{p}$ and $\alpha$ characterize the magnitudes of specific strain energy and gradient energies with respect to the chemical driving force. $\phi$ is defined as $4 G \varepsilon_{0}{ }^{2} /|\Delta f|$ $[22,26]$, where $G$ is the shear modulus, $\varepsilon_{0}$ is the largest component in the misfit strain tensor between $\alpha_{2}$ and $\gamma$, and $\Delta f$ is the chemical driving force. According to [27], the shear modulus $G$ decreases with increasing temperature from $103.5 \mathrm{GPa}$ at $298 \mathrm{~K}$ to 94.8 $\mathrm{GPa}$ at $723 \mathrm{~K}$. By linear extrapolation, $G$ is assumed to be $62.4 \mathrm{GPa}$ at $1373 \mathrm{~K}$. 
According to [28], $\varepsilon_{0}$ is 0.03 . The chemical driving forces considered in this paper are 360, 450 and $540 \mathrm{~J} / \mathrm{mol}$, which correspond to 34,43 and $51 \times 10^{6} \mathrm{~J} / \mathrm{m}^{3}$, respectively. Therefore the $\phi$ value should be in the range of 4.4 to 11 under these conditions. Since the effect of elastic energy contribution has been investigated in our previous study [7], the $\phi$ value is set to a fixed value of 10 throughout the current study. The gradient energy coefficients $\beta_{i j}^{p}$ are assumed to be variant independent and only diagonal terms are non-zero. According to Veeraraghavan et al [29], the energy of the disordered unfaceted $\alpha / \gamma$ interfaces typically ranges from 300 to $600 \mathrm{~mJ} / \mathrm{m}^{2}$, while the energy of the coherent or partially coherent faceted interfaces ranges from 25 to 200 $\mathrm{mJ} / \mathrm{m}^{2}$. These values yield an anisotropy parameter, $\beta_{11}\left(\beta_{22}\right) / \beta_{33}$, ranging from 3 to 12 . From our simulations it is found that the width to thickness ratio of the lamella obtained with $\beta_{11}\left(\beta_{22}\right) / \beta_{33}=5$ agrees with what was observed experimentally [30], thus, $\beta_{11}\left(\beta_{22}\right) / \beta_{33}=5$ (i.e., $\left.\alpha=\beta_{11}=\beta_{22}=5 \beta_{33}\right)$ is chosen in all simulations presented in this study. The value of $v$ determines whether the growth of nucleus is diffusion or interface controlled and it is assumed to be 1, which means it is mainly interface controlled. $c_{1}$ and $c_{2}$ are chosen to be 0.38 and 0.48 respectively, which are the equilibrium $\mathrm{Al}$ concentrations in the $\alpha_{2}$ and $\gamma$ phases, respectively, at $1100^{\circ} \mathrm{C}$, this temperature is $25^{\circ} \mathrm{C}$ below the eutectoid temperature, which yields a chemical driving force of $450 \mathrm{~J} / \mathrm{mol}$ according to the thermodynamic database used in the current study and the mean composition of the alloy is assumed to be 0.46 . All the Voigt elastic constants are zero except that: $C_{11}=C_{22}=175 \mathrm{GPa} ; C_{33}=220 \mathrm{GPa} ; C_{12}=88.7 \mathrm{GPa}$; $C_{13}=C_{23}=62.3 \mathrm{GPa} ; C_{44}=C_{55}=62.2 \mathrm{GPa} ; C_{66}=\left(C_{11}-C_{12}\right) / 2$ [31]. Periodic 
boundary condition is imposed along all three dimensions.

\section{Simulation results}

\subsection{Nucleation and evolution of $\gamma$ precipitates under normal stresses}

Experimentally, most HIPping operations are carried out under stress levels of 300 MPa or below, e.g. 130 [32], 175 [33], 207 [12], 275 [34] and 300 MPa [35]. Stress levels of $125,175,275,325,375$ and $425 \mathrm{MPa}$ are considered in our simulations for different types of external stresses for this parametric study. The chemical driving force considered is $450 \mathrm{~J} / \mathrm{mol}$. In TiAl alloys, all lamellae formed during the $\alpha \rightarrow \gamma+\alpha_{2}$ transformation are parallel to the basal plane of the matrix phase, and the stress applied along the X $[11 \overline{2} 0]$ and Y [ $\overline{1} 100]$ will be referred to as "along" or "parallel" to the basal plane of the matrix or lamellar interface, while along the Z [0001] will be referred to as "perpendicular" to the basal plane or lamellar interface. The applied stresses include compression or tension along $\mathrm{Z}$ (ComZ or TenZ), compression or tension along $\mathrm{X}$ (ComX or TenX), and isostatic pressing (ISP). The effect of shears along both $0^{\circ}$ (the negative shear (shearN) indicates the right part of the matrix shear upward [ $\overline{1} 100]$ relative to the left part) and $180^{\circ}$ (the positive shear (shearP $\left.[1 \overline{1} 00]\right)$ is the reversal of shearN) are discussed in section 3.2.

The typical spatial distribution and particle morphology of $\gamma$ precipitates during the nucleation stage at reduced time $\tau=1$ (real time $t=\tau \times 10^{3} \mathrm{~s}$ ) under $125 \mathrm{MPa}$ compression along the $X[11 \overline{2} 0]$ are shown in Fig. 1(a). Most precipitates formed consist of twin related variants, as marked by the arrows. When the random noise is 
turned off after $\tau=1$, some small precipitates disappear and the rest grow. Finally only some prevailing precipitates survive and form multi-twin lamellae. The typical microstructures at different stages are shown in Figs. 1(b) to (f).

Fig. 2(a) shows the evolution of composition distribution along the direction perpendicular to the laths during the transformation. The aluminum concentration of initial $\gamma$ precipitates $\left(c_{\mathrm{i}}\right)$ is about 0.55 . It decreases as the $\gamma$ phase grows. When most matrix $\alpha$ transforms to $\gamma$, the aluminum concentration in the residual $\alpha$ decreases, and this will promotes the transformation of $\alpha$ to $\alpha_{2}$. Finally, the concentrations of $\gamma$ and $\alpha_{2}$ phases approach their equilibrium values of 0.47 and 0.38 , respectively. The volume fraction of $\alpha_{2}$ decreases as $\gamma$ grow with time, and finally go steady to about 0.1 after $\tau$ $=4.2$, in agreement with thermodynamic estimation. As shown in Fig. 2(b), the equilibrium concentration of $\alpha_{2}$ and $\gamma$ phases are determined by the common tangent of the two free energy curves, point $c_{1}$ and $c_{2}$ on line 1 . However the $c_{\mathrm{i}}$ can only be determined by the equilibrium with a starting composition of $\alpha$ phase as the average composition of the alloy $\left(c_{\mathrm{a}}\right)$, utilizing the equal partial free energy or chemical potential of $\mathrm{Al}$ (defined as the derivative of the free energy to the $\mathrm{Al}$ concentration) in the two phase. The $c_{\mathrm{i}}$ determined this way is shown in figure 2(b), as the cutting point of line 2' with the free energy curve of $\gamma$ phase. The obtained $c_{\mathrm{i}}$ agree with our phase field simulation.

Two parameters are introduced to describe the microstructure character, i.e., the twin area fraction (TAF) and volume fraction (VF). The TAF is defined as total twin boundary area over total $\gamma / \gamma$ boundary area. VF is the ratio of total volume of 
precipitates to the system volume. The TAFs are calculated at different moments from the simulation results obtained under different types and levels of external normal stresses. The results are shown in Fig. 3. The TAFs are nearly $100 \%$ in the early nucleation stage at $\tau<0.6$, which indicates that all multi-layer precipitates consist of twin-related variants from either correlated or collective nucleation [7] under the influence of elastic interactions. When $0.6<\tau<1$, the TAFs decrease quickly because of the impingement of the growing precipitates that leads mostly to the formation of non-twin related boundaries. From the moment when the random noises are turned off at $\tau=1$ to the moment when $\tau \sim 2.2$, the TAFs may increase or decrease, depending on the interplay between the dissolution of small precipitates and the growth and impingement of large precipitates. During $2.2<\tau<4.2$, the TAFs decrease sharply because the survived precipitates grow and impinge upon each other quickly, forming large amount of non-twin related interfaces. After $\tau \sim 4.2$, the TAFs change slowly and reach more or less constant after $\tau \sim 7$ when lamellar microstructures are fully developed, and the volume fraction of the product phase $(\gamma)$ is larger than 0.9 .

The TAF at the final stage of the simulation is about $70 \%$ for stress-free condition, as shown in Fig. 3(a). It is within the range of some experimental observations of $67-77 \%$ [36] and 72.4\% [37]. The influence of different types of external stresses on the TAF can be seen clearly from Fig. 3(a). The TAF increases significantly under ComZ and TenX while it decreases slightly under isostatic compression, ComX and TenZ. In the end, the TAF under ComZ is $17 \%$ larger than that in the absence of external stress, while the TAF under TenZ is $5 \%$ smaller. The effect of stress amplitude on TAF 
variation is shown in Figs. 3(b) to (d). Stress along the $Y$ axis is not discussed in this work because its effects are similar to that caused by external stress along the $\mathrm{X}$ due to the similar strain components, Fig. 3(a).

The volume fractions (VFs) of different variant $\left(\mathrm{V}_{1}\right.$ to $\left.\mathrm{V}_{6}\right)$ at $\tau=1$ and their summation under different levels of compression (negative) and tension (positive) along $\mathrm{X}$ and Z, and isostatic pressing are shown in Figs. 4(a), (b) and (c), respectively. When the external stress is applied along $\mathrm{X}$, the VF of every variant decreases monotonically when the stress changes continuously from compression to tension (Fig. 4(a)), while the opposite is true when the external stress is applied along Z (Fig. 4(b)). The effect of isostatic pressing on the VF is relatively small (Fig. 4(c)).

The above simulations are carried out with the chemical driving force of $450 \mathrm{~J} / \mathrm{mol}$ ( $\Delta f$ shown in Fig. $2(\mathrm{~b})$ corresponds to $\mathrm{c}=0.46$ and at $1100^{\circ} \mathrm{C}$ ). It should be noted that the twin area fraction also varies with the chemical driving force [7]. The larger the chemical driving force, the smaller the TAF. The TAFs as functions of both applied stress level and chemical driving force level, 360, 450 and $540 \mathrm{~J} / \mathrm{mol}$ (corresponds to different temperature for the alloy with a concentration $c=0.46$ ), are shown in Figs. 5(a), (b) and (c), respectively for tension and compression along $\mathrm{X}$ and $\mathrm{Z}$, and isostatic pressing at $\tau=9$. It can be seen that the TAF increases significantly under tension along $\mathrm{X}$ (i.e., parallel to the lamellar interface) and compresion along $\mathrm{Z}$ (i.e., prependicular to the lamellar interface), decreases slightly under compression along $\mathrm{X}$ and tension along $\mathrm{Z}$, and remains more or less constant under isostatic pressing. For example, under the medium chemical driving force $(450 \mathrm{~J} / \mathrm{mol})$ the TAF increases 
from $70 \%$ (witout any external load) to $84 \%$ under $375 \mathrm{MPa}$ tension along $\mathrm{X}$, decreases from $70 \%$ (under no load) to $68 \%$ under $375 \mathrm{MPa}$ compression along X. With the increase in chemical driving force, the TAF decreases and the effects from all the applied stresses are reduced. For example, the range of TAF under different levels of stress along $\mathrm{X}$ is roughly $70-88 \%$ when the chemical driving force is relatively small (360 J/mol), and decreases to $60-69 \%$ when the chemical driving force increases to $540 \mathrm{~J} / \mathrm{mol}$.

\subsection{Nucleation and evolution of $\gamma$ precipitates under shear stresses}

The above simulations are carried out under normal stresses. We now consider shear stresses. The volume fractions of different variants and their total volume fraction as functions of levels of negative $(\mathrm{N},[\overline{1} 100])$ and positive $(\mathrm{P},[1 \overline{1} 00])$ shear stresses at reduced time $\tau=1$ are shown in Fig. 6 . The volumes of twin related variants are very close to each other if there is no external load (Fig. 6 at $0 \mathrm{MPa}$ ). A negative shear is found to promote the formation of $\mathrm{V}_{1}, \mathrm{~V}_{4}$ and $\mathrm{V}_{6}$, and suppress $\mathrm{V}_{2}, \mathrm{~V}_{3}$ and $\mathrm{V}_{5}$, while a positive shear is found to have just the opposite effects. This is because the positive shear applied is along the direction of the transformation shear of $\mathrm{V}_{2}$ and in the reverse direction of that of $\mathrm{V}_{1}$. At the same time, the transformation shear direction of $\mathrm{V}_{3}$ and $\mathrm{V}_{5}$ is closer to $\left(60^{\circ}\right)$ the positive shear applied, compared with $\mathrm{V}_{4}$ and $\mathrm{V}_{6}$ $\left(120^{\circ}\right)$. Thus under positive shear, $V_{2}$ is promoted the most, $V_{3}$ and $V_{5}$ second, and $V_{1}$ is suppressed the most, $\mathrm{V}_{4}$ and $\mathrm{V}_{6}$ second.

The total volume fraction of $\gamma$ precipitates increases while the TAFs decrease 
monotonously during the precipitate growth under shear (Fig. 7), regardless of the direction of shear. The TAFs approach zero at $\tau=3.4$ when the shear stress exceeds $375 \mathrm{MPa}$. Under large shear, the unfavored variants in any twinning pair will be consumed finally by shear transformation into its twin-related neighbor variants, and the TB between them will disappear. Therefore the thickness of the laths will be increased by shear, and the total number of the laths decreased compared to that without a stress.

Such shear deformation may occur in extrusion or rolling process in the two phase field, especially at the outer part of the extruded rod and near the surface of the rolled sheet. For grains with orientations favoring shear along lamellar plane, the $\gamma$ transformation and detwinning of some variants may be promoted, forming certain textures containing fewer variants. Depending on the amplitude of applied stress, such shear induced transformation may be coupled with lamellar fragmentation and recrystallization, as observed experimentally during extrusion of a rectangular billet [38].

\section{Discussions}

The above simulations show that isostatic pressing promotes $\gamma$ nucleation during the $\alpha$ $\rightarrow \gamma+\alpha_{2}$ phase transformation, as shown in Fig. 4(c). This agrees with the experimental observation where the HIPping suppresses the dissolution kinetics of $\gamma$ into $\alpha$ [12]. A similar pressure-assisted phase transformation has also been found in $\beta / \mathrm{B} 2 \rightarrow \gamma+\alpha_{2}$ transformation, where the $\beta / \mathrm{B} 2$ phase becomes unstable during HIPping 
and shows a strong tendency to transform to a more closed packed $\gamma+\alpha_{2}$ structure [39]. These results are not surprising because the compression promotes the volume decreasing phase transformation and suppresses the volume increasing ones. The average atomic volume of $\gamma$ phase $\left(16.3 \times 10^{-30} \mathrm{~m}^{3}\right)$ is smaller than that of the $\alpha / \alpha_{2}$ phase $\left(16.7 \times 10^{-30} \mathrm{~m}^{3}\right)$ or the $\beta / \mathrm{B} 2$ phase $\left(17.3 \times 10^{-30} \mathrm{~m}^{3}\right)$ in Ti-46Al-2Cr-2Mo-0.25Si $-0.3 \mathrm{~B}\left(\right.$ at. \%) at the HIPping temperature (about $1000{ }^{\circ} \mathrm{C}$ ) [39].

The high twin area fraction shown in Figs. 3 and 5 is attributed partly to the elastic strain energy relaxation. The distributions of interaction energies between a pre-existing and nucleating precipitates in 3-D space are shown in Fig. 8. The position and size of the pre-existing plate $\left(\mathrm{V}_{1}\right)$ is shown by the dashed circle. The calculations indicate that the most negative elastic interaction energy exists between twin related variants, Fig. 8(b). Though the negative elastic interaction energy exists between ordered-domain related variants (Figs. 8(c) and (e)), the negative value regions are small compared to that of twin related variants. The minimum is $-1.0 \times 10^{5} \mathrm{~J} / \mathrm{m}^{3}$, significantly smaller than that between the two twin related variants $\left(\operatorname{MinI}_{\mathrm{T}}=\right.$ $\left.-1.2 \times 10^{5} \mathrm{~J} / \mathrm{m}^{3}\right)$

Furthermore, the twin area fraction changes under different types of applied stresses are shown in Fig. 3, 5 and 7. The changes of interaction energy extrema under 275 MPa of different types of stresses are summarized in Table 2. All extrema show slight increases under ComZ and TenX as compared to those in the absence of external stresses, and slight decreases under isostatic pressing, ComX and TenZ. $\operatorname{MinI}_{\mathrm{T}}$ and MinI $_{\mathrm{OD}}$ decrease while MaxI $\mathrm{I}_{\mathrm{SELF}}$ and MaxI $\mathrm{I}_{\mathrm{PT}}$ increase under shearP. The effect of 
shearN on the interaction energy is just opposite to that of shearP. The relative magnitude of interaction energy extrema changes under applied stress. For example, the ratio of interaction energy minimum between twin related variants $\left(\mathrm{MinI}_{\mathrm{T}}\right)$ and ordered-domain related variants $\left(\mathrm{MinI}_{\mathrm{OD}}\right)$ under $\mathrm{ComZ}$ is slightly larger than that without applied stress, i.e., $\left(\operatorname{MinI}_{\mathrm{T}} / \mathrm{MinI}_{\mathrm{OD}}\right)_{\mathrm{ComZ}}>\left(\mathrm{MinI}_{\mathrm{T}} / \mathrm{MinI}_{\mathrm{OD}}\right)_{\mathrm{No}}$, as shown in Table 2. This means that the interaction between twin related variants becomes stronger relatively under ComZ, so twin related nucleation is more preferred in this condition, and therefore, higher twin area fraction will be obtained, see Figs. 3(a) and $5(b)$.

Based on the simulations and discussions above, the changes of twin frequency in TiAl alloys induced by the applied stress during thermo-mechanical processing or by the residual stress after that should be considered. In fully lamellar polycrystals, the twin frequency in different grains should be more or less alike theoretically if the $\alpha \rightarrow$ $\gamma+\alpha_{2}$ transformation take places in the absence of external stress. While when the alloy is under thermo-mechanical processing such as flat rolling, the stress magnitude and orientation are generally different from the surface to interior in the plate during rolling [40] and so is the residual stress after the processing [41]. Therefore, the stress state is different from one grain to another, and the twin frequency of different grains after phase transformation should be diverse under these stresses.

Taking advantage of the effect that the twin frequency can be altered by external stress, the twin frequency refinement through stress control during phase transformation is possible. But the difficulty is that the grains in the matrix should 
have similar orientations at least. Fortunately, the grain orientation can be modified through heat flux control during casting or some thermo-mechanical processes. For TiAl alloy bars after $\alpha$ field hot extrusion, most $c$ axis of grains tend to be perpendicular to the extrusion direction [42]. TiAl alloy bars extruded through a rectangular die in the two phase field and then solution treated below $\alpha$ transus exhibit a dominant texture with the $c$ axis parallel to the transverse direction [38]. After extrusion, compression perpendicular to the basal plane of the matrix applied during the $\alpha \rightarrow \gamma+\alpha_{2}$ phase transformation may be beneficial to improving the twin frequency.

In this simulation, the homogeneous modulus appoximation is used for simplicity, i.e., both the parent and product phases have the same elastic constants. Therefore, the mismatch between them will not change under applied stress. Although with inhomogeneous molulus model [43], the soft phase will have slightly larger strain under applied stress and more accurate elastic strain energy will be obtained, acceptable results can be obtained fast and easily with the present model. The interaction energy calculation with pre-existing precipitates under applied stresses shows mainly the effect of applied stresses on the autocatalytic transformation rather than that on variant selection during nucleation. Quantitative characterization of the latter requires a combination of the phase field simulation with the free-end NEB method [44], with supercritical particles obtained in the phase field simulations being used as the end images.

The macro-strain from the transformation shear differs from system to system. In the 
case of Co-Ni disordered alloy, only one variant can be formed by any combination of the three lattice shift on the same plane, different macro-strain may be induced by different combination of partial slips, depending on the composition and condition [45, 46]. While for $\alpha \rightarrow \gamma+\alpha_{2}\left(\mathrm{HCP} \rightarrow \mathrm{L}_{0}+\mathrm{D}_{19}\right)$ transformation in TiAl alloys, the stacking sequence $\mathrm{ABABAB}$ of $\mathrm{HCP}$ becomes either $\mathrm{ABCABC}$ or $\mathrm{ACBACB}$ through different shear. Atomic ordering takes place at the same time, so that one of the six variants can be formed by only one shear. Experimental study in TiAl alloys has shown that [47], surface relief can be formed during transformation. This provides direct experimental evidence in support of the displacive nature of $\alpha \rightarrow \gamma+\alpha_{2}$ transformation with macro-strain. In this investigation, the partial dislocations forming one variant have the same Burgers vector, therefore relatively large transformation strain is involved. Small variants may disappear due to elastic strain energy and interfacial energy minimization. With further evolution, one variant may occupy the whole lath due to the small system size along $\mathrm{X}$ and $\mathrm{Y}$ direction. While for the larger systems simulated, two or more different variants may exist within one lamella, with interfaces roughly perpendicular to the lamella formed between them, although the area fraction is small compared with the interface between different layers.

The heterogeneous nucleation from various defects may have significant influence on the final microstructure, as shown experimentally that the stacking faults formed from quenching or deformation can serve as nucleation sites for $\gamma$ plates and induce fine lamellar structure [48], however, due to the complexity of deformed microstructure, 
the detailed dislocation or stacking fault structure during high temperature deformation is practically unknown to us, therefore, only homogeneous nucleation is considered in the present simulations. The present simulation is valid only in the elastic deformation range, or the former deformation induced defect structure is extremely random that it can be described by the random noise used in the simulation here.

Plastic deformation can be effective in generating dislocations to promote precipitates nucleation and microstructure control [49]. Furthermore, the lamellar structure may also be tailored by controlling the dislocation distribution taking advantage of nanoscale plastic deformation produced by nano-imprinting, nano-indentation and nano-grooving [50]. However, the phase field models taking into account of the plastic deformation [51] and dislocation [15] are out of the scope of the present work and will be discussed separately.

\section{Conclusions}

The effects of applied stresses on $\gamma$ nucleation and evolution in TiAl alloys during $\alpha$ $\rightarrow \gamma+\alpha_{2}$ phase transformation have been investigated through elastic interaction energy calculations and phase field simulations. It is found that:

(1) Among the six types of interactions, the most negative elastic interaction energy exists between twin related variants in the absence of external stress. Compression perpendicular and tension parallel to the basal plane raise all interaction energies; while tension perpendicular and compression parallel to the basal plane or isostatic 
pressing make them lower.

(2) The variant selection due to autocatalytic nucleation may be enhanced or suppressed by applied stresses. Compression perpendicular and tension parallel to the basal plane reduce the overall nucleation rate and promote twin formation, while isostatic pressing, compression parallel, tension perpendicular to the basal plane does the opposite. Shears parallel to the basal plane always increase the overall nucleation rate, but reduce twin boundary area fraction.

(3) The influence of external stress on twin area fraction is compared to that of chemical energy change for phase transformation. The twin area fraction increases $15 \%$ under $275 \mathrm{MPa}$ compression perpendicular to the basal plane, while such increase can be obtained equally without stress by a chemical driving force reduction of $180 \mathrm{~J} / \mathrm{mol}$.

(4) For a highly textured sample where most grains have similar basal plane orientations, compression perpendicular to the basal plane applied during $\alpha \rightarrow \gamma+\alpha_{2}$ phase transformation is beneficial to increasing lamellar twin boundary area fraction.

\section{Acknowledgement}

The financial support of the Ministry of Science and Technology of China (2011CB606404), the National Science Foundation of China (51171195 and 50911130367) (D.S.X. and R.Y.), the National Science Foundation (CMMI-0728069 and DMR1008349) (Y.W) and China Scholarships Council (2008608034) (C.Y.T) and Shenyang Supercomputing Center of CAS are gratefully acknowledged.

\section{References}

[1] Liu CT, Schneibel JH, Maziasz PJ, Wright JL, Easton DS. Tensile properties and fracture 
toughness of TiAl alloys with controlled microstructures. Intermetallics 1996;4:429.

[2] Kim YW. Strength and ductility in TiAl alloys. Intermetallics 1998;6:623.

[3] Kishida K, Inui H, Yamaguchi M. Deformation of PST crystals of a TiAl/Ti ${ }_{3} \mathrm{Al}$ two-phase alloy at $1000^{\circ} \mathrm{C}$. Intermetallics 1999;7:1131.

[4] Parthasarathy TA, Subramanian PR, Mendiratta MG, Dimiduk DM. Phenomenological observations of lamellar orientation effects on the creep behavior of Ti-48at.\%Al PST crystals. Acta Mater 2000;48:541.

[5] Kim HY, Maruyama K. Stability of lamellar microstructure of hard orientated PST crystal of TiAl alloy. Acta Mater 2003;51:2191.

[6] Gibson MA, Forwood CT. Slip transfer of deformation twins in duplex $\gamma$-based Ti-Al alloys. Part I. Transfer across $\gamma-\gamma$ coherent twin interfaces. Philos Mag A 2000;80:2747.

[7] Teng CY, Zhou N, Wang YZ, Xu DS, Du A, Wen YH, Yang R. Phase field simulation of twin boundary fractions in fully lamellar TiAl alloys. Acta Mater 2012;60:6372.

[8] Xu WC, Shan DB, Zhang H, Li XA, Zhang YZ, Nutt S. Effects of extrusion deformation on microstructure, mechanical properties and hot workability of $\beta$ containing TiAl alloy. Mater Sci Eng A 2013;571:199.

[9] Liu CT, Maziasz PJ. Microstructural control and mechanical properties of dual-phase TiAl alloys. Intermetallics 1998;6:653.

[10] Schillinger W, Bartels A, Gerling R, Schimansky FP, Clemens H. Texture evolution of the $\gamma$ - and the $\alpha / \alpha_{2}$-phase during hot rolling of $\gamma$-TiAl based alloys. Intermetallics 2006;14:336.

[11] Bystrzanowski S, Bartels A, Stark A, Gerling R, Schimansky FP, Clemens H. Evolution of microstructure and texture in Ti-46Al-9Nb sheet material during tensile flow at elevated temperatures. 
Intermetallics 2010;18:1046.

[12] Zhao L, Beddoes J, Morphy D, Wallace W. Microstructure and mechanical properties of a PM TiAl-W alloy processed by hot isostatic pressing. Mater Sci Eng A 1995;192/193:957.

[13] Karthikeyan S, Mills MJ. The role of microstructure stability on compression creep of fully lamellar $\gamma$-TiAl alloys. Intermetallics 2005;13:985.

[14] Wang YZ, Chen LQ. Simulation of microstructural evolution using the field method. In: Kaufmann EN, editor. Methods in Materials Research. New York: New York, 2000. p.2a.3.1.

[15] Wang YZ, Li J. Phase Field Modeling of Defects and Deformation. Acta Mater 2010;58:1212.

[16] Shen C, Simmons JP, Wang YZ. Effect of elastic interaction on nucleation: I. Calculation of the strain energy of nucleus formation in an elastically anisotropic crystal of arbitrary microstructure. Acta Mater 2006;54:5617.

[17] Zhou N. Simulation study of directional coarsening (rafting) of $\gamma^{\prime}$ in single crystal Ni-Al. The Ohio State University; The Department of Materials Science and Engineering, vol. Doctor Thesis. Columbus: The Ohio State University, 2008. p.46.

[18] Shen C, Simmons JP, Wang YZ. Effect of elastic interaction on nucleation: II. Implementation of strain energy of nucleus formation in the phase field method. Acta Mater 2007;55:1457.

[19] Schuster JC, Palm M. Reassessment of the binary Aluminum-Titanium phase diagram. J Phase Equilib Diff 2006;27:255.

[20] Blackburn MJ. Some aspects of phase transformations in titanium alloys. In: Jaffee RI, Promisel NE, editors. The Science, Technology and Applications of Titanium. Oxford: Pergamon Press, 1970. p.633.

[21] Wen YH, Chen LQ, Hazzledine PM, Wang YZ. A three-dimensional phase-field model for 
compter simulation of lamellar structure formation in $\gamma \mathrm{TiAl}$ intermetallic alloys. Acta Mater $2001 ; 49: 2341$.

[22] Wen YH, Wang Y, Bendersky Y, Chen LQ. Microstructural evolution during the $\alpha_{2}-\alpha_{2}+\mathrm{O}$ transformation in Ti-Al-Nb alloys: phase-field simulation and experimental validation. Acta Mater $2000 ; 48: 4125$.

[23] Khachaturyan AG. Theory of Structural Transformations in Solids. New York: Wiley, 1983.

[24] Jin YM, Wang YU, Khachaturyan AG. Three-dimensional phase field microelasticity theory of a multivoid multicrack system in an elastically anisotropic body: model and computer simulations. Philos Mag 2008;83:1587.

[25] Shen C, Wang Y. Coherent precipitates-phase field method. In: Sidney Y, editor. Handbook of meterials modeling. Heidelberg Springer Netherlands, 2005. p.2117.

[26] Artemev A, Wang Y and Khachaturyan AG. Three-dimensional phase field model and simulation of martensitic transformation in multilayer systems under applied stresses. Acta mater 2000;48:2503.

[27] He Y, Schwarz RB, Darling T, Hundley M, Whang SH and Wang ZM. Elastic constants and thermal expansion of single crystal $\gamma$-TiAl from 300 to 750 K. Mater Sci Eng A 1997;239-240:157.

[28] Grinfeld MA, Hazzledine PM, Shoykhet B and Dimiduk DM. Coherency stresses in lamellar Ti-Al. Metall Mater Trans A 1998;29A:937.

[29] Veeraraghavan D, Wang P, Vasudevan VK. Nucleation kenetics of the $\alpha$ to $\gamma_{M}$ massive transformation in a Ti-47.5 at\% Al alloy. Acta Mater 2003; 51:1721.

[30] Zghal S, Thomas M and Couret A. Structural transformations activated during the formation of the lamellar microstructure of TiAl alloys. Intermetallics 2005;13:1008.

[31] Tanaka K, Okamoto K, Inui H, Minonishi Y, Yamaguchi M, Koiwa M. Elastic constants and their 
temperature dependence for the intermetallic compound $\mathrm{Ti}_{3} \mathrm{Al}$. Philosophical Magazine $\mathrm{A}$ $1996 ; 73: 1475$.

[32] Rahaman MN, Dutton RE, Semiatin SL. Fabrication of dense thin sheets of $\gamma$-TiAl by hot isostatic pressing of tape-cast monotapes. Mater Sci Eng A 2003;360:169.

[33] Zheng RT, Cheng GA, Li XJ, Cao GX, Fu LF, Zhang YG, Chen CQ. The relationship between fracture toughness and microstructure of fully lamellar TiAl alloy. J Mater Sci 2007;42:1251.

[34] Suryanarayana C, Sundaresan R, Froes FH. TiAl formation by mechanical alloying. Mater Sci Eng A 1992;150:117.

[35] Suryanarayana C, Al-Aqeeli N. Mechanically alloyed nanocomposites. Prog Mater Sci 2013;58:383.

[36] Zghal S, Thomas M, Naka S, Couret A. Migration of ordered domain boundaries and its effect on the lamellar interfaces in Ti-Al-based alloys. Philos Mag Lett 2001;81:537.

[37] Zghal S, Naka S, Couret A. A quantitative TEM analysis of the lamellar microstructure in TiAl based alloys. Acta Mater 1997;45:3005.

[38] Liu RC, Liu D, Tan J, Cui YY, Yang R, Liu FY, Withey PA. Textures of rectangular extrusions and their effects on the mechanical properties of thermo-mechanically treated, lamellar microstructure, Ti-47Al-2Cr-2Nb-0.15B. Intermetallics 2014;52:110.

[39] Zhang D, Dehm G, Clemens H. Effect of heat-treatments and hot-isostatic pressing on phase transformation and microstructure in a $\beta /$ B2containing $\gamma$-TiAl based alloy. Scripta mater 2000;42:1065. [40] Kocich R, Machackova A, Fojtik F. Comparison of strain and stress conditions in conventional and ARB rolling process. Int J Mech Sci 2012;64:54.

[41] Hidveghy J, Michel J, Bursak M. Residual stress in microalloyed steel sheet. Metalurgija 
2003;42:103.

[42] Appel F, Oehring M, Paul JDH, Klinkenberg C, Carneiro T. Physical aspects of hot-working gamma-based titanium aluminides. Intermetallics 2004;12:791.

[43] Zhou N, Shen C, Mills MJ, Wang Y. Contributions from elastic inhomogeneity and from plasticity to $\gamma^{\prime}$ rafting in single-crystal Ni-Al. Acta Mater 2008;56:6156.

[44] Shen C, Li J, Wang Y. Finding critical nucleus in solid-state transformations. Metall Mater Trans A 2008;39:976.

[45] Waitz T, Karnthaler HP. The f.c.c. to h.c.p. martensitic phase transformation in CoNi studied by TEM and AFM methods. Acta Mater 1997;45:837.

[46] Liu YN, Yang H, Liu Y, Jiang BH, Ding J, Woodward R. Thermally induced fcc $\leftrightarrow$ hcp martensitic transformation in Co-Ni. Acta Mater 2005;53:3625.

[47] Sun YQ, Surface relief and the displacive transformation to the lamellar microstructure in TiAl. Philos Mag Lett 78;1998:297.

[48] Karadge M, Gouma PI. A structural aspect of $\alpha\left(\alpha_{2}\right) \rightarrow$ lamellar $\alpha_{2}+\gamma$ transformation in $\gamma$-TiAl. Philos Mag Lett 2004;84:451.

[49] Koizumi Y, Fujita T, Minamino Y, Hata S. Effects of plastic deformation on lamellar structure formation in Ti-39 at.\% Al single crystals. Acta Mater 2010;58:1104.

[50] Wei DX, Koizumi Y, Nishiyama H, Yamanake A, Yoshino M, Miyamoto S, Yoshimi K, Chiba A. Nanoplastic deformation on Ti-39 at.\% Al single crystals for manipulation of every single $\gamma$ lamella. Acta Mater 2014;76:331.

[51] Gaubert A, Bouar YL, Finel A. Coupling phase field and viscoplasticity to study rafting in Ni-based superalloys. Philos Mag 2010;90:375. 
Table 1. Boundary types between different $\gamma$ variants.

\begin{tabular}{ccl}
\hline Group $\gamma_{\mathrm{I}}$ & Group $\gamma_{\mathrm{II}}$ & \multicolumn{1}{c}{ Relationships } \\
\hline $\mathrm{V}_{1}$ & $\mathrm{~V}_{2}$ & twin $\longrightarrow 180^{\circ}$ \\
$\mathrm{V}_{3}$ & $\mathrm{~V}_{4}$ & twin $\longrightarrow 180^{\circ}$ \\
$\mathrm{V}_{5}$ & $\mathrm{~V}_{6}$ & twin $\longrightarrow 180^{\circ}$ \\
ordered 4 & ordered 4 & pseudo \\
domain $\downarrow 120^{\circ}$ & domain $\downarrow_{120^{\circ}}$ & -twin
\end{tabular}

The horizontal double headed arrows indicate that the pairs of variants in the same row are twin related while the vertical double headed arrows indicate that ordered-domain boundary formed between any of the two variants in the same column when they are in contact. The cross of double headed arrows indicate that pseudo-twin boundary formed between any of the two variants in column I and II (from different rows) when they are in contact.

Table 2. Changes in the extrema $\left(\mathrm{J} / \mathrm{m}^{3}\right)$ of interaction energy between a pre-existing $\left(\mathrm{V}_{1}\right)$ and nucleating precipitates $\left(\mathrm{V}_{1}-\mathrm{V}_{6}\right.$, respectively), under $275 \mathrm{MPa}$ of different types of external stresses relative to those in the absence of external stress whose values are listed in the column "NoStress".

\begin{tabular}{lcccccccc}
\hline & ShearP & ComZ & TenX & NoStress & ISP & ComX & TenZ & ShearN \\
\hline MaxI $_{\text {SELF }}$ & 267 & 59 & 24 & 142141 & -12 & -26 & -60 & -266 \\
MinI $_{\mathrm{T}}$ & -250 & 55 & 24 & -123692 & -11 & -23 & -55 & 250 \\
MinI $_{\mathrm{OD}}$ & -149 & 66 & 45 & -105853 & -13 & -45 & -66 & 150 \\
MaxI $_{\mathrm{PT}}$ & 152 & 67 & 46 & 106937 & -13 & -46 & -68 & -152 \\
\hline
\end{tabular}




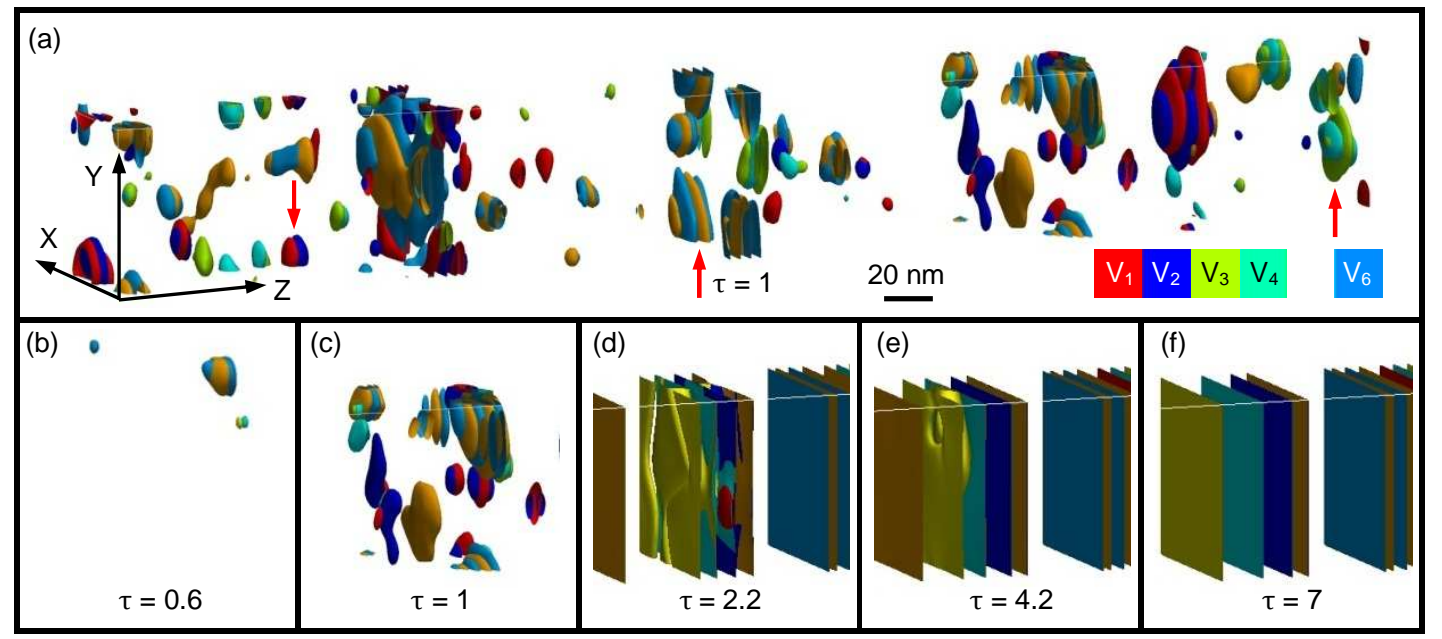

Fig. 1. Typical precipitate distribution and morphology at reduced time $\tau=1$ (a) and their evolution at different stages ( $\mathrm{b}$ - f). All multi-layer precipitates consist of twin related variants; typical configuration is marked by arrows. Different colors stand for different variants and the $\alpha / \alpha_{2}$ phase are made invisible.
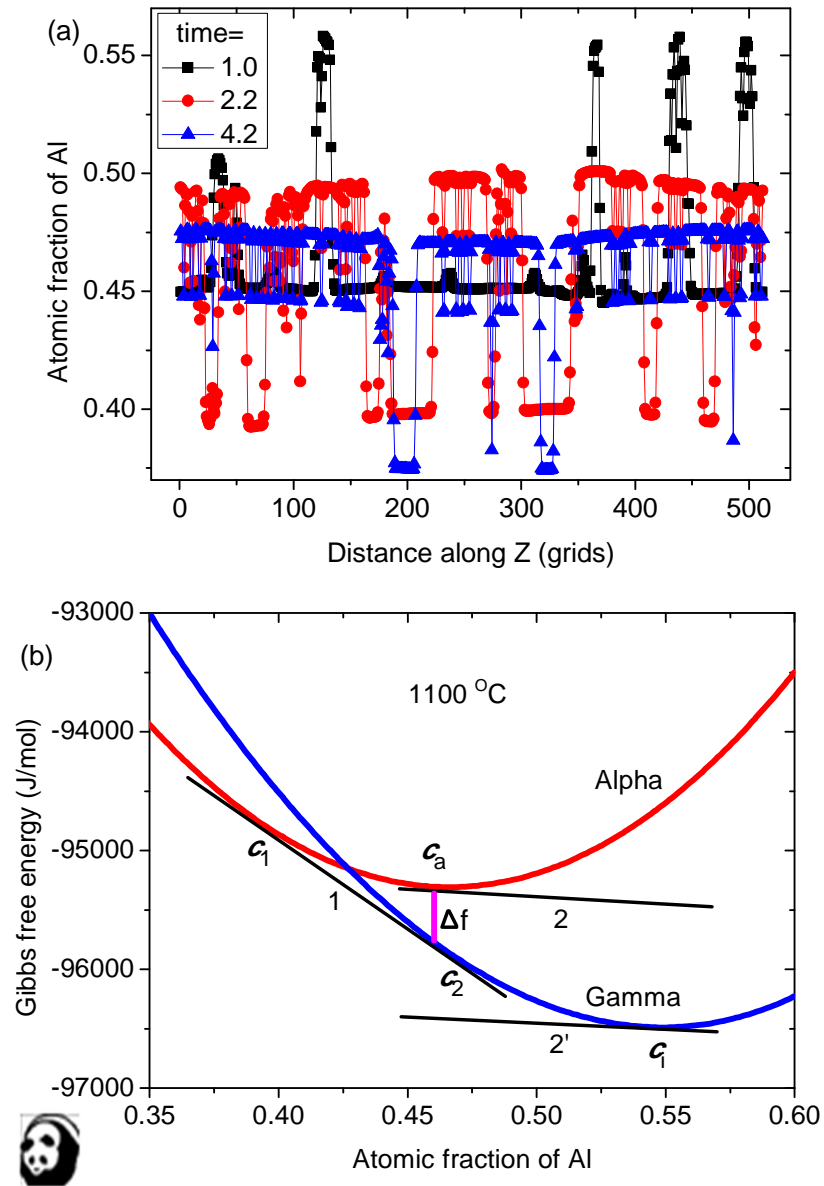

Fig. 2. (a) The concentration profiles along the direction perpendicular to the lamellar plane at different reduced time of 1, 2.2 and 4.2, respectively. (b) The Gibbs free energy curves of $\alpha$ and $\gamma$ phases at $1100^{\circ} \mathrm{C}$. Line 1 is the common tangent of free energy curves of $\alpha$ and $\gamma$. The $c_{1}$ and $c_{2}$ stand for the equilibrium concentration of $\alpha_{2}$ and $\gamma$ phase. The $c_{\mathrm{a}}$ stands for the average aluminum concentration of the alloy. The line 2 is the tangent of the free energy curve of $\alpha$ through $c_{\mathrm{a}}$. Line 2' which parallel to the line 2 is the tangent of free energy curve of $\gamma$. The cutting point $c_{\mathrm{i}}$ indicates the concentration of initial precipitate of $\gamma$ phase. 

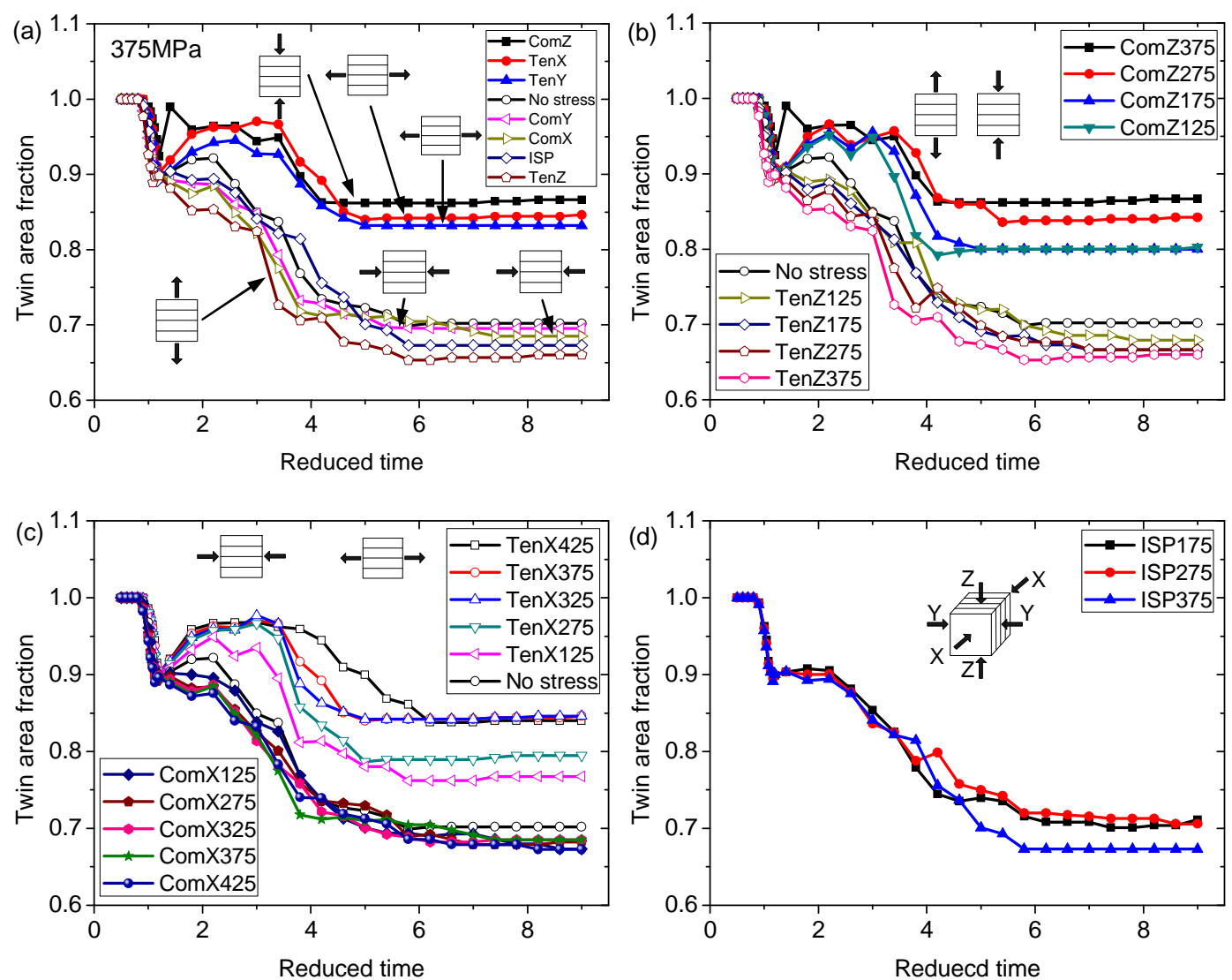

Fig. 3. (a) Comparison of the TAF evolution under tension, compression and zero stress. TAF evolutions under stress of different level (MPa) along $\mathrm{Z}(\mathrm{b})$ and $\mathrm{X}$ (c), and under isostatic pressing (d). The stress direction relative to the basal/lamellar plane is shown schematically. 

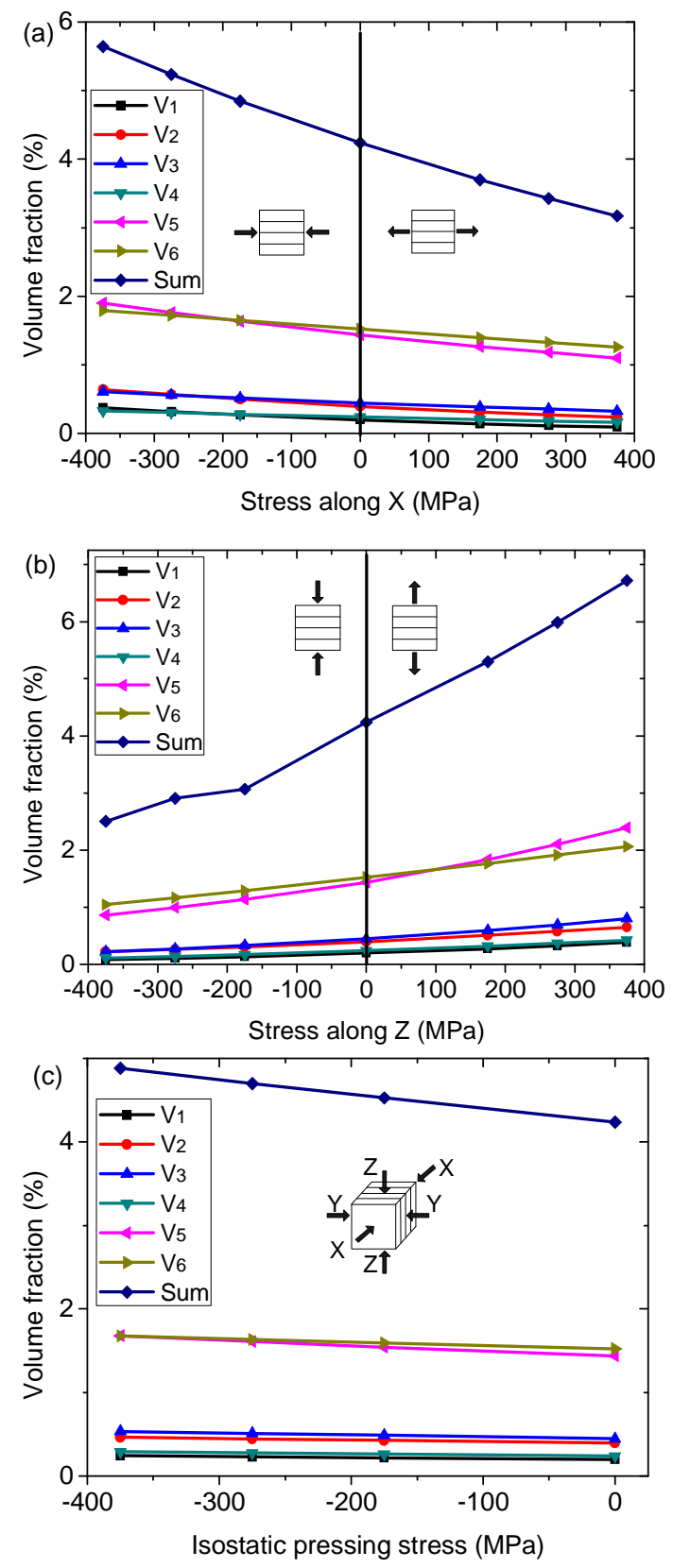

Fig. 4. Volume fractions of $V_{1}$ to $V_{6}$ at $\tau=1$ and their summation under different levels of compression (negative) and tension (positive) along $\mathrm{X}$ (a), Z (b) and isostatic pressing (c). 

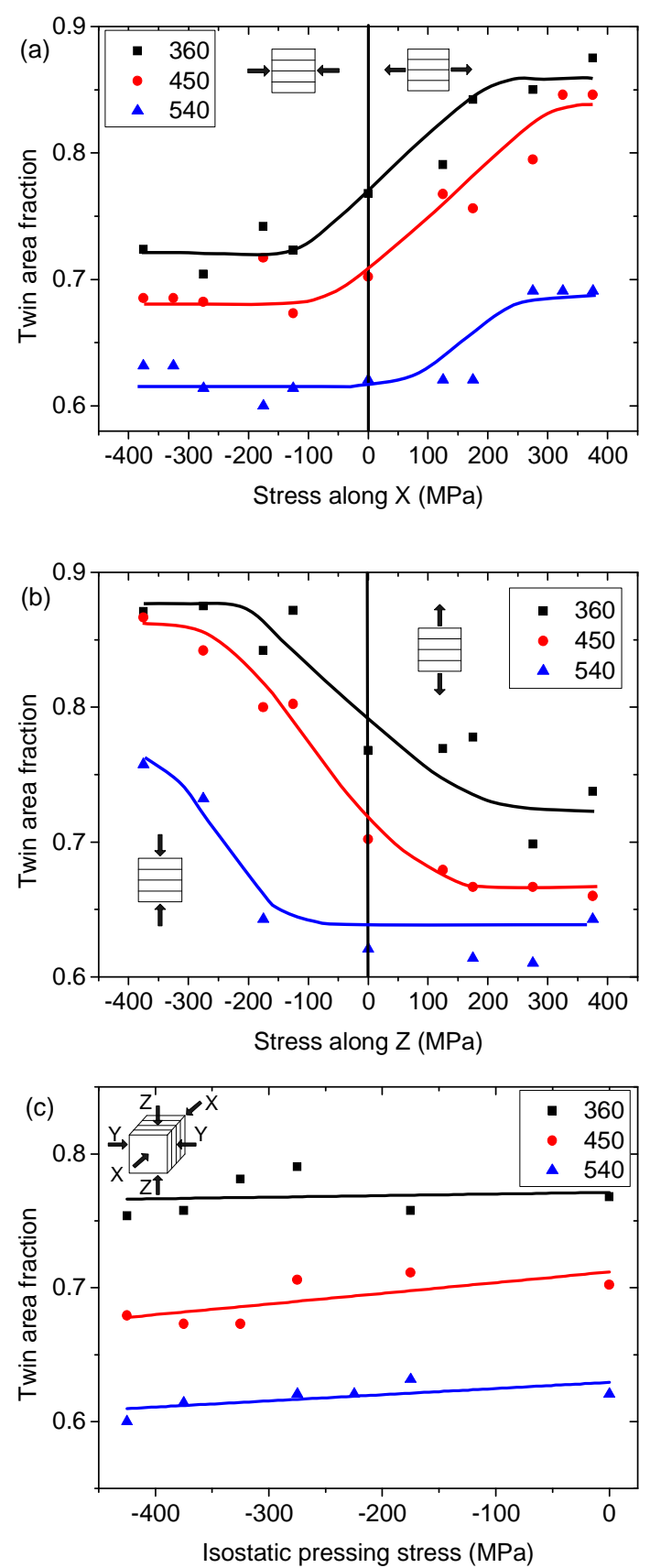

Fig. 5. TAFs under different levels of chemical driving force $(\mathrm{J} / \mathrm{mol})$ and external stress along $X$ (a), Z (b) and isostatic pressing (c) at reduced time $\tau=9$. 


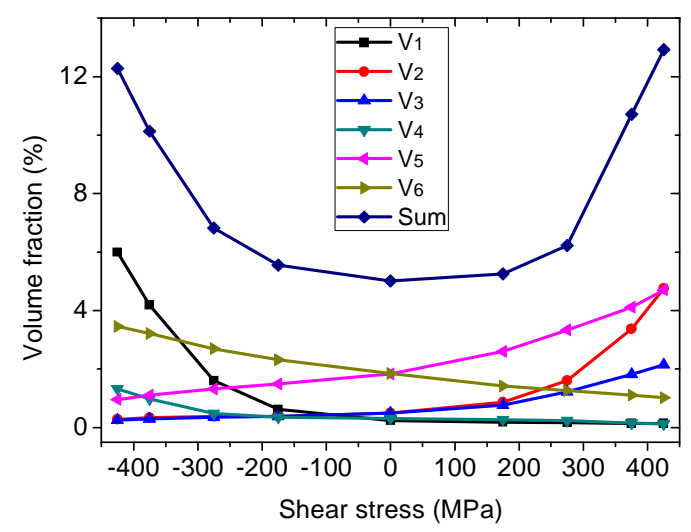

Fig. 6. The volume fractions of the six variants and their summation obtained at reduced time $\tau=$ 1 as functions of external shear stresses.
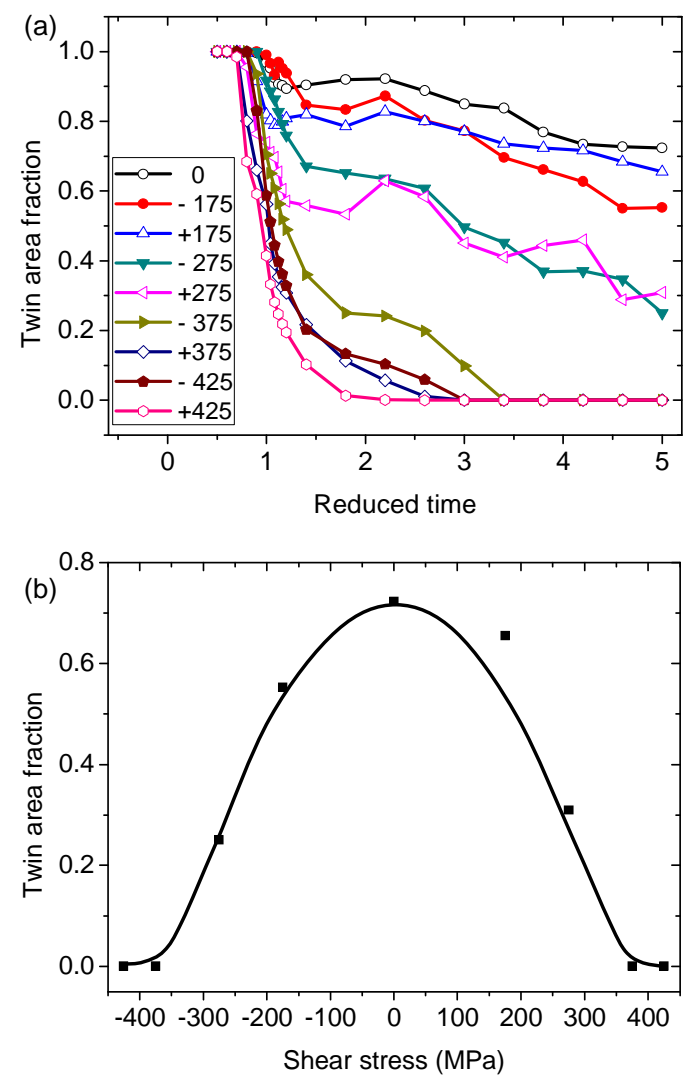

Fig. 7. Comparison of TAF evolution (a) and TAF at reduced time $\tau=5$ (b) under different shear (MPa). 


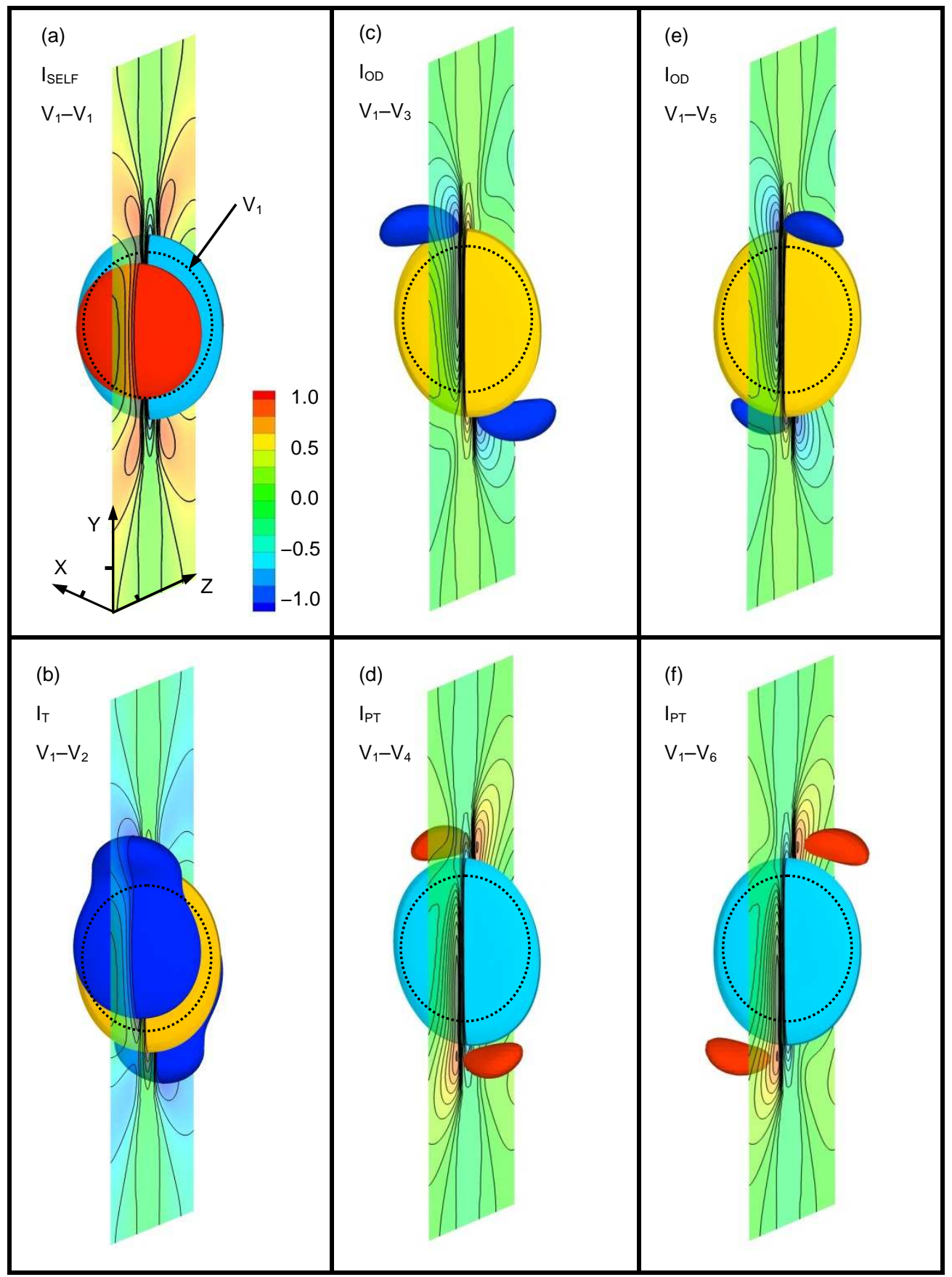

Fig. 8. 3-D iso-surfaces and contour plot (central cross section) of interaction energies between a pre-existing plate $\mathrm{V}_{1}$ (shown with arrow in (a) and outlined by the dashed lines in (a) - (f)) and nucleating precipitates $\left(V_{1}\right.$ to $V_{6}$, respectively). The interaction energy between the same variants $\left(\mathrm{V}_{1}-\mathrm{V}_{1}\right.$ in (a)) is denoted by $\mathrm{I}_{\mathrm{SELF}}$, and those between twin $\left(\mathrm{V}_{1}-\mathrm{V}_{2}\right.$ in (b)), pseudo-twin $\left(\mathrm{V}_{1}-\mathrm{V}_{4}\right.$, $\mathrm{V}_{1}-\mathrm{V}_{6}$ in (d) and (f)) and ordered-domain $\left(\mathrm{V}_{1}-\mathrm{V}_{3}, \mathrm{~V}_{1}-\mathrm{V}_{5}\right.$ in (c) and (e)) related variants are denoted by $\mathrm{I}_{\mathrm{T}}, \mathrm{I}_{\mathrm{PT}}$ and $\mathrm{I}_{\mathrm{OD}}$, respectively. The numbers of contour legend shall be multiplied by $10^{5}\left(\mathrm{~J} / \mathrm{m}^{3}\right)$. The scale on the $\mathrm{X}, \mathrm{Y}$ and $\mathrm{Z}$ axis in (a) is $10 \mathrm{~nm}$, respectively. 